\title{
Universal finite-sample effect on the perturbation growth in chaotic dynamical systems
}

\author{
Hiroya Nakao* and Shuya Kitada \\ Department of Physics, Graduate School of Science, Kyoto University, Kyoto 606-8502, Japan \\ Alexander S. Mikhailov \\ Abteilung Physikalische Chemie, Fritz-Haber-Institut der Max-Planck-Gesellschaft, Faradayweg 4-6, 14195 Berlin, Germany
}

(Received 28 March 2006; published 28 August 2006)

\begin{abstract}
The finite-sample effect on the growth of moments of the perturbation observed in numerical simulations of chaotic dynamical systems is studied. To numerically estimate the moments, only a limited number of sample trajectories can be utilized, and therefore the moments exhibit pure exponential growth only initially, and give way to relaxed growth thereafter. Such transition is a consequence of the unobservability of rare events in finite sample sets. Using the large-deviation formalism for chaotic time series, we estimate the relaxation time and derive the post-relaxation growth law. We demonstrate that even after the relaxation, each moment still obeys a universal growth law of different type, which reflects physical information on the statistics of chaotic expansion rates.
\end{abstract}

DOI: 10.1103/PhysRevE.74.026213

PACS number(s): 05.45.-a, 02.50.-r

\section{INTRODUCTION}

In understanding chaotic dynamical systems, fluctuations in the expansion rate of a small perturbation to the original orbit are essential [1-13]. Generalized Lyapunov exponents, which represent average growth rates of the moments of the perturbation at various orders, are useful in assessing temporal fluctuations in the expansion rate. Their spectrum characterizes the fluctuating expansion rate at various scales continuously as a function of the order of the moment. Universal statistics of the expansion rate can also be characterized using the rate function of the finite-time Lyapunov exponent, which measures the average expansion rate of the perturbation in a finite time interval. Generalized Lyapunov exponents are related to the rate function of the finite-time Lyapunov exponent through the large-deviation formalism $[2-7,14,15]$.

In numerically estimating the generalized Lyapunov exponents, we solve a linearized equation describing the dynamics of a small perturbation, and measure the exponential growth rates of its moments at various orders. Usually, generalized Lyapunov exponents depend nonlinearly on its order. This dependence is the signature of intermittent behavior, where rare fluctuations in the expansion rate tend to dominate the evolution of the moments, while the majority of fluctuations give only a small contribution [16-20].

It is known that reliable numerical measurement of the generalized Lyapunov exponents is rather difficult, because a large number of sample trajectories is needed to obtain good statistics, especially for high-order moments [3]. As we demonstrate in this paper, in actual numerical calculations, growth of the moments is strongly affected by the number of sample trajectories that can be used in the calculation. True intermittent exponential growth of each moment can be observed only initially, and shortly gives way to a relaxed growth law. If we measure the generalized Lyapunov expo-

\footnotetext{
*Corresponding author: nakao@ton.scphys.kyoto-u.ac.jp
}

nents after this transition, they exhibit nonintermittent behavior, which is completely different from the intermittent one measured initially.

In this paper, we investigate such finite-sample effect on the moment growth by using an argument similar to the one we previously used in analyzing the finite-size saturation of field variables in a stochastic autocatalytic system with diffusion [20]. The dynamics of small perturbations is modeled as a random multiplicative process driven by chaotic multipliers. Using the large-deviation formalism for the chaotic expansion rates, we analytically estimate the relaxation time of the moments and their growth laws after the relaxation. We demonstrate that even after the relaxation, each moment obeys a universal nonexponential growth law determined by the statistics of the chaotic expansion rates, so that they still provide physical information about the chaotic system under consideration. We verify our estimates numerically using low-dimensional chaotic dynamical systems.

\section{STATISTICS OF EXPANSION RATES}

In this section, we summarize the large-deviation (or thermodynamic-multifractal) formalism of the chaotic expansion rates for temporally discrete dynamical systems. The case of continuous dynamical systems is discussed later.

\section{A. Dynamics of a small perturbation}

Let us consider a discrete chaotic dynamical system

$$
\mathbf{x}(t+1)=\mathbf{F}(\mathbf{x}(t)),
$$

where $t$ represents the time step, $\mathbf{x}$ the state vector, and $\mathbf{F}$ its dynamics. A small perturbation vector $\mathbf{q}(t)$ to the original orbit $\mathbf{x}(t)$ obeys

$$
\mathbf{q}(t+1)=J_{F}(\mathbf{x}(t)) \mathbf{q}(t),
$$

where $J_{F}$ denotes the Jacobian matrix of $\mathbf{F}$. Assuming the perturbation vector to be aligned with the direction of the maximal expansion rate, we focus on its amplitude 


$$
q(t)=|\mathbf{q}(t)|,
$$

whose dynamics is described by

$$
q(t+1)=\exp \{\eta(t)\} q(t) .
$$

Here, $\eta(t)$ represents the instantaneous expansion rate of the orbit

$$
\eta(t)=\ln \left\{\frac{q(t+1)}{q(t)}\right\},
$$

which fluctuates randomly due to the chaotic dynamics of the original orbit $\mathbf{x}(t)$ (this definition of the expansion rate follows Ref. [10]). If we consider Eq. (4) as a stochastic process, the dynamics of $q(t)$ is a discrete-time random multiplicative process driven by the chaotic noise $\eta(t)$, which is generally non-Gaussian and colored. Hereafter, we assume the ergodicity and stationarity of the chaotic processes under consideration.

\section{B. Growth exponents}

For simplicity, we choose the initial condition of $q(t)$ as $q(0)=1$. The ordinary Lyapunov exponent is given by the average of $\ln q(t)$ as

$$
h=\lim _{t \rightarrow \infty} \frac{1}{t}\langle\ln q(t)\rangle .
$$

Here and hereafter, $\langle\cdots\rangle$ denotes average over the natural invariant measure of the chaotic system under consideration $[2,3]$.

To characterize the temporal fluctuations in the expansion rate, we also consider the $k$ th order moment $\left\langle q(t)^{k}\right\rangle$, where $k$ is real and positive. $\left\langle q(t)^{k}\right\rangle$ generally grows exponentially as

$$
\left\langle q(t)^{k}\right\rangle \simeq \exp \{\lambda(k) t\}
$$

so that its growth rate is defined as

$$
\lambda(k)=k h(k)=\lim _{t \rightarrow \infty} \frac{1}{t} \ln \left\langle q(t)^{k}\right\rangle .
$$

Here, $h(k)=\lambda(k) / k$ is the $k$ th order generalized Lyapunov exponent (or entropy spectrum) [1-4]. When $k$ is small, the ordinary Lyapunov exponent is recovered because

$$
\ln \left\langle q(t)^{k}\right\rangle=\ln \langle\exp (k \ln q(t))\rangle \simeq \ln \langle 1+k \ln q(t)\rangle \simeq k\langle\ln q(t)\rangle,
$$

so that in the limit $k \rightarrow 0$ we obtain

$$
\lim _{k \rightarrow 0} h(k)=\lim _{t \rightarrow \infty} \frac{1}{t}\langle\ln q(t)\rangle=h .
$$

It is convenient to consider the $k$ th order relative moment $\left\langle q(t)^{k}\right\rangle /\langle q(t)\rangle^{k}$ in assessing the intermittency of $q(t)$ [16-20]. The relative moment also grows exponentially as

$$
\frac{\left\langle q(t)^{k}\right\rangle}{\langle q(t)\rangle^{k}} \simeq \exp \{\Lambda(k) t\}
$$

whose growth rate is defined as

$$
\Lambda(k)=\lim _{t \rightarrow \infty} \frac{1}{t} \ln \frac{\left\langle q(t)^{k}\right\rangle}{\langle q(t)\rangle^{k}}=\lambda(k)-k \lambda(1) .
$$

We call this growth rate the "intermittency exponent." When $\Lambda(k)$ vanishes, the temporal sequence of $q(t)$ is nonintermittent; moments at different orders behave similarly. On the other hand, when $\Lambda(k)$ is nonzero, $q(t)$ is termed intermittent. In this case, higher-order moments grow more rapidly than lower-order moments, which indicates that rare realizations of the expansion rate play dominant roles in determining the moments.

For example, if the probability density function (PDF) $P(q, t)$ of $q(t)$ evolves self-similarly with a single scaling exponent $\alpha$ as

$$
P(q, t)=e^{-\alpha t} f\left(e^{-\alpha t} q\right),
$$

the $k$ th order moment $\left\langle q(t)^{k}\right\rangle$ grows as

$$
\left\langle q(t)^{k}\right\rangle \simeq e^{k \alpha t},
$$

so that the relative moment does not grow,

$$
\frac{\left\langle q(t)^{k}\right\rangle}{\langle q(t)\rangle^{k}} \simeq 1 .
$$

Therefore, in this case, we obtain

$$
\lambda(k)=\alpha k, \quad \Lambda(k)=0 .
$$

Namely, the growth rate $\lambda(k)$ depends linearly on $k$, the intermittency exponent $\Lambda(k)$ is always zero, and the generalized Lyapunov exponent $h(k)$ takes a constant value $\alpha$. Thus, the evolution of $q(t)$ is nonintermittent.

However, in general, the PDFs of random multiplicative processes, including the growth of perturbation vectors in chaotic dynamical systems, do not evolve self-similarly. Therefore, $\lambda(k)$ becomes a nonlinear function of $k$, and $\Lambda(k)$ does not vanish. The evolution of $q(t)$ cannot be characterized by a single scaling exponent in such cases.

\section{Rate function}

Fluctuations in the expansion rate can also be characterized by the "rate function" of the finite-time Lyapunov exponent. In the large-deviation formalism of chaotic time series, $q(t)$ is decomposed into individual "events," $\exp (u t)$. From Eq. (4), we obtain

$$
q(t)=q(0) \exp (u t), \quad u=\frac{1}{t} \sum_{s=0}^{t-1} \eta(s),
$$

where $u$ is the finite-time Lyapunov exponent of $q(t)$ in a time interval $t$. Since $u$ is a mean value of $t$ random numbers $\eta(s)$, the PDF of $u$ at large $t$ is expected to obey $[2-7,9,11-15]$

$$
P(u, t) \sim \exp \{-t S(u)\},
$$

where $S(u)$ is called a rate function, a convex function that takes the minimum value 0 at the true mean value of $u=h$ (at the ordinary Lyapunov exponent). The probability to observe the events with $u \neq h$ decreases exponentially with $t$, so that 
they are called "rare events." Using the saddle approximation (or the Laplace's method), the moment at large $t$ can be estimated as

$$
\begin{aligned}
\left\langle q(t)^{k}\right\rangle & \simeq \int\{\exp (u t)\}^{k} P(u, t) d u \\
& =\int \exp \{k u-S(u)\} t d u \\
& \simeq \exp \left\{k u^{*}(k)-S\left(u^{*}(k)\right)\right\} t,
\end{aligned}
$$

where $u^{*}(k)$ satisfies

$$
\frac{d}{d u}\{k u-S(u)\}=0 .
$$

This event $u^{*}(k)$ dominates the $k$ th moment $\left\langle q(t)^{k}\right\rangle$. Thus, the growth rate $\lambda(k)$ is represented using the rate function $S(u)$ through a Legendre transform as

$$
\lambda(k)=\max _{u}\{k u-S(u)\} .
$$

Conversely, $S(u)$ is represented using $\lambda(k)$ as

$$
S(u)=\max _{k}\{k u-\lambda(k)\} .
$$

Since $S(u)$ is a convex function, moments corresponding to $k>0$ are determined by the events with $u>h$, and $u^{*}(k)$ is an increasing function of $k$. Therefore, higher-order moments are determined by the events with larger values of $u$, which are more rare.

If the central limit theorem holds for the temporal sequence of $\eta(t), S(u)$ can be approximated by a quadratic curve around its minimum as [14,15]

$$
S(u) \simeq \frac{S^{\prime \prime}(h)}{2}(u-h)^{2},
$$

as we illustrate later for the modified Bernoulli map and for the Duffing model. In chaotic dynamical systems, however, $\eta(t)$ may not always obey the central limit theorem, for example, near the bifurcation points where the temporal correlation of $\eta(t)$ becomes anomalously long [5-7,11-13]. In such cases, $S(u)$ cannot be approximated quadratically, as we illustrate later for the logistic map and for the Lorenz model. The rate function $S(u)$ and the generalized Lyapunov exponent $\lambda(k)$ give complementary characterizations of the statistics of the fluctuating expansion rates.

\section{FINITE-SAMPLE EFFECT}

In this section, using one-dimensional maps as examples, we demonstrate that numerically estimated moments exhibit true exponential growth initially, and quickly relax due to the finite-sample effect. The case of continuous dynamical systems is discussed later.

\section{A. Numerical estimation of the moments}

We first describe the numerical procedure that we use in the calculation of the Lyapunov exponents here. As we dem- onstrate below, numerical estimates of the moments of the perturbation are found to depend strongly on the number of sample trajectories used in the calculation.

In numerically calculating the ordinary Lyapunov exponent, we replace the average of the logarithm of the small perturbation over the natural invariant measure $\langle\ln q(t)\rangle$ by the average over $N$ sample trajectories starting from $N$ random initial conditions

$$
[\ln q(t)]=\frac{1}{N} \sum_{i=1}^{N} \ln q_{i}(t),
$$

where $[\cdots]$ denotes the sample average. Usually, this quantity increases linearly with $t$, whose slope yields the estimate of the ordinary Lyapunov exponent. As long as we use sufficient number of trajectories, e.g., $N=100$, it does not suffer from the finite-sample effect, namely, $[\ln q(t)]$ approximates $\langle\ln q(t)\rangle$ nicely without significantly depending on $N$. This is because the nonlinear logarithmic transformation tends to ignore the fluctuation in $q(t)$.

Similarly, in numerically estimating the generalized Lyapunov exponent, we first calculate the sample average of $q(t)^{k}$ using $N$ sample trajectories as

$$
\left[q(t)^{k}\right]=\frac{1}{N} \sum_{i=1}^{N} q_{i}(t)^{k},
$$

where $i$ denotes the $i$ th realization of $q(t)$. Although the slope of $\ln \left[q(t)^{k}\right]$ gives the generalized Lyapunov exponent $\lambda(k)$ in principle, $\ln \left[q(t)^{k}\right]$ strongly fluctuates even if we use rather large $N$, e.g., $N=1000$, especially for large $k$ and $t$. This is a consequence of the strong intermittency of $q(t)$ arising from its random multiplicative dynamics. Therefore, we further average $\ln \left[q(t)^{k}\right]$ over $M$ sample averages as

$$
\overline{\ln \left[q(t)^{k}\right]}=\frac{1}{M} \sum_{j=1}^{M} \ln \left[q(t)^{k}\right]_{j},
$$

where the overline denotes the average over the realizations of the logarithm of the sample average $\ln \left[q(t)^{k}\right]$. The subscript $j$ represents the $j$ th realization of the sample average $\left[q(t)^{k}\right]$. In the following, we consider this quantity as an estimator of the (logarithm of) true ensemble-averaged moment $\ln \left\langle q^{k}\right\rangle$. Though not explicitly shown, we should keep in mind that this quantity depends on $N$ and $M$.

As we discuss below, the growth law of $\overline{\ln \left[q(t)^{k}\right]}$ coincides with the true growth law of $\ln \left\langle q^{k}\right\rangle$ only in the short period just after the onset of growth. After this period, the growth property of the numerical moment significantly deviates from the true one. The evolution of $\overline{\ln \left[q(t)^{k}\right]}$ essentially depends on the number of sample trajectories $N$, whereas an increase in the number of averages $M$ only suppresses its fluctuations. Even without the second averaging $(M=1)$, the result is qualitatively unchanged, though it becomes quite noisy.

\section{B. One-dimensional chaotic map}

We first consider a one-dimensional chaotic map described by 


$$
x(t+1)=f(x(t)) .
$$

A small perturbation $q(t)$ to the orbit $x(t)$ obeys a linearized equation

$$
q(t+1)=\left|f^{\prime}(x(t))\right| q(t),
$$

so that the instantaneous expansion rate is simply given by

$$
\eta(t)=\ln \left|f^{\prime}(x(t))\right|
$$

\section{Modified Bernoulli map}

As the first example, let us consider the modified Bernoulli map [1]

$$
f(x)=\left\{\begin{array}{l}
\frac{x}{p} \quad(0 \leq x \leq p), \\
\frac{x-p}{1-p} \quad(p \leq x \leq 1),
\end{array}\right.
$$

which is the simplest chaotic map with a fluctuating expansion rate. The instantaneous expansion rate $\eta(t)$ takes only two values $\ln (1 / p)(0 \leq x \leq p)$ and $\ln \{1 /(1-p)\}(p \leq x \leq 1)$. We fix the parameter at $p=0.75$ in the following numerical simulations. Since the invariate density $\rho(x)$ of this modified Bernoulli map is constant $\rho(x) \equiv 1$, its ordinary Lyapunov exponent is given by

$$
h=p \ln \frac{1}{p}+(1-p) \ln \frac{1}{1-p},
$$

which yields $h \simeq 0.562$ at $p=0.75$.

Figures 1(a) and 1(b) display the growth of the moments and the relative moments obtained numerically at several values of $k$. In the numerical calculations, it was not necessary to renormalize the perturbation to avoid numerical overflow. Since the Lyapunov exponent of this map is relatively small, and also the exponential growth of the moments of the linearized perturbation is drastically slowed down due to finite-sample effect, the numerical moments are well within the allowed range of the computer throughout our numerical calculations. In the very early period, each moment grows exponentially. Each relative moment also grows exponentially, indicating the intermittent growth of the moments. As shown in Fig. 1(c), the growth exponent $\lambda(k)$ measured in this early period depends nonlinearly on $k$. In this case, $\lambda(k)$ can be calculated analytically as [1]

$$
\lambda(k)=\ln \left\{p^{1-k}+(1-p)^{1-k}\right\},
$$

which is also shown in the figure for comparison. In Fig. $1(d)$, we can see that the intermittency exponent $\Lambda(k)$ is clearly nonzero. Correspondingly, the generalized Lyapunov exponent $h(k)$ shown in Fig. 1(e) is not constant. Only its asymptotic value for $k \rightarrow 0$ coincides with the ordinary Lyapunov exponent $h$.

However, unlike $[\ln q(t)]$, exponential growth of each numerical moment $\overline{\ln \left[q(t)^{k}\right]}$ quickly relaxes, and gives way to a different growth law after this initial period, in contradiction to our intuition. Though this transition is not so clear in Fig.
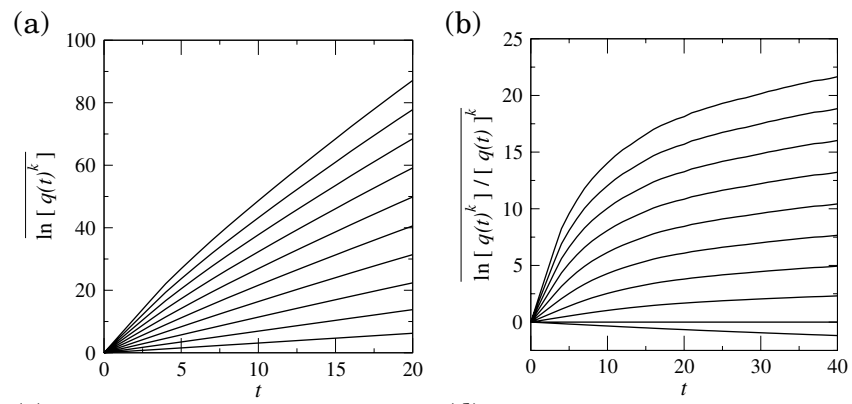

(c)

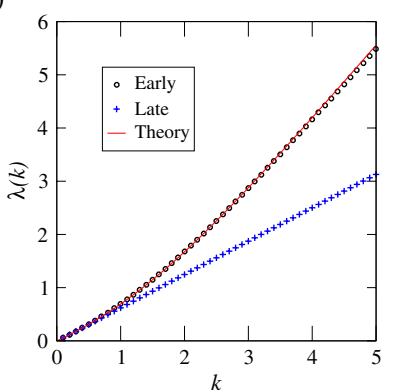

(d)
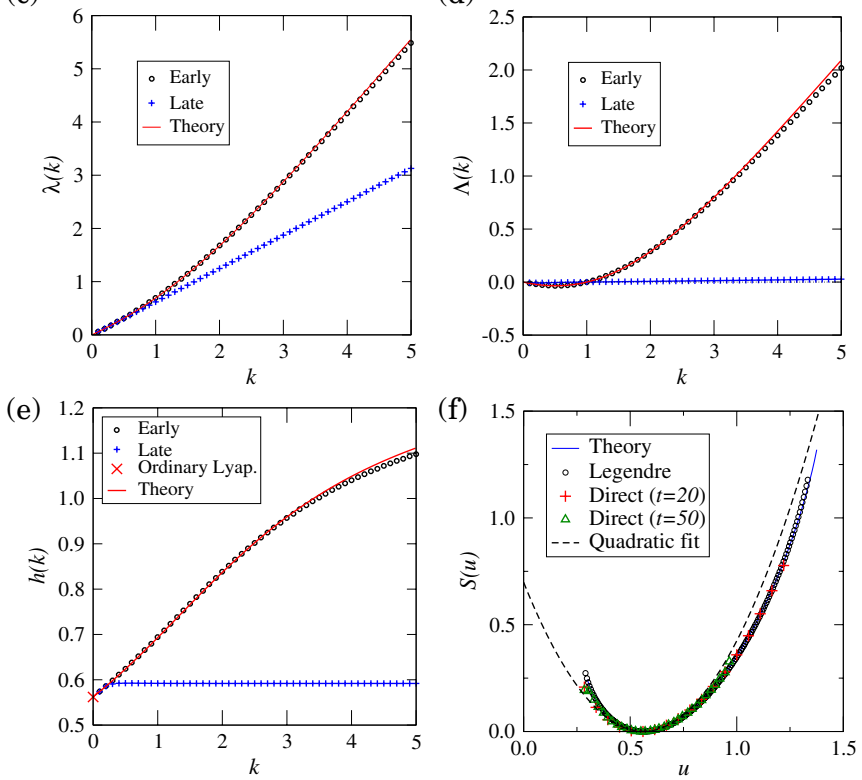

FIG. 1. (Color online) Results for the modified Bernoulli map. The number of samples is $N=1000$, and the number of averages is $M=1000$. (a),(b) Growth of $\overline{\ln \left[q(t)^{k}\right]}$ and $\overline{\ln \left\{\left[q(t)^{k}\right] /[q(t)]^{k}\right\}}$ at several values of $k$ (the bottom curve corresponds to $k=0.5$, and the top curve to 5.0, with curves in intervals of 0.5 in between). (c),(d),(e) Growth rate $\lambda(k)$, intermittency exponent $\Lambda(k)$, and generalized Lyapunov exponent $h(k)$. Values measured in the initial period and those measured after the onset of relaxation are shown. Theoretical curves are also plotted for comparison. In (e), the ordinary Lyapunov exponent $h$ corresponding to the $k \rightarrow 0$ limit is also shown. (f) Rate function $S(u)$ of the finite-time Lyapunov exponent $u$. The theoretical curve, the Legendre transform of the numerically obtained $\lambda(k)$, direct numerical calculations using time intervals of $t=20$ and $t=50$, and the quadratic fit around the minimum are plotted.

1(a), it can clearly be recognized in Fig. 1(a), where the numerical relative moments stop showing linear exponential growth quickly and exhibit relaxed growth. If we measure $\lambda(k)$ in this late regime, we obtain nonintermittent exponents that depend linearly on $k$ as shown in Fig. 1(c). Correspondingly, $\Lambda(k)$ almost vanishes, and $h(k)$ takes almost a constant value close to the ordinary Lyapunov exponent $h$ as shown in Figs. 1(d) and 1(e). As we discuss later, the growth law of the relaxed moment is not purely exponential but also contains a stretched-exponential component. The small discrepancy between $h(k)$ and $h$ is due to the numerical errors caused by such a nonexponential component. 
The rate function $S(u)$ of the modified Bernoulli map can analytically be calculated [1,9], which is shown in Fig. 1(f). It is expressed parametrically as

$$
\begin{aligned}
& S(\psi)=\psi \ln \frac{\psi}{p}+(1-\psi) \ln \frac{1-\psi}{1-p}, \\
& u(\psi)=\psi \ln \frac{1}{p}+(1-\psi) \ln \frac{1}{1-p},
\end{aligned}
$$

where the parameter $\psi$ satisfies $0 \leq \psi \leq 1$. In the figure, curves obtained by the Legendre transform of numerically measured $\lambda(k)$ in the early period, and by direct numerical calculations using time intervals of $t=20$ and $t=50$, are also shown. The finite-time Lyapunov exponent $u$ takes values continuously between $\ln (1 / p)$ and $\ln \{1 /(1-p)\}$. $S(u)$ takes its minimum at the ordinary Lyapunov exponent, $u=h$, and the curvature at the minimum is given by

$$
S^{\prime \prime}(h)=\left\{p(1-p)\left(\ln \frac{1}{p}-\ln \frac{1}{1-p}\right)^{2}\right\}^{-1},
$$

which yields $S^{\prime \prime}(h) \simeq 4.42$ at $p=0.75$. As shown in the figure, this gives a reasonable fit around $u=h$, indicating that the central limit theorem holds for $\eta(t)$.

\section{Logistic map}

As another example, we consider the logistic map

$$
f(x)=1-2 x^{2} .
$$

We fix the parameter at the fully chaotic value 2 throughout our discussion. In this case, $\eta(t)$ takes values continuously between $-\infty$ and $\ln 4$. The ordinary Lyapunov exponent is given by

$$
h=\ln 2 .
$$

Figures 2(a) and 2(b) show the growth of $\overline{\ln \left[q(t)^{k}\right]}$ and $\ln \left\{\left[q(t)^{k}\right] /[q(t)]^{k}\right\}$ estimated numerically at several values of $k$. In the numerical calculations, we renormalized the perturbation every step by the ordinary Lyapunov exponent $h$ $=\ln 2$ and compensated the numerical results after taking the logarithm to avoid numerical overflow of the higher order moments. As in the case of the modified Bernoulli map, each moment grows exponentially for small $t$, and thereafter its growth law undergoes a change. Each relative moment grows exponentially only initially, and then approaches a constant value. Compared with the modified Bernoulli map, the relaxation is even steeper. Figures 2(c)-2(e) display $\lambda(k)$, $\Lambda(k)$, and $h(k)$ measured in the early and in the late period. $\lambda(k)$ of the logistic map can also be obtained analytically [6,7], which is comprised of two straight lines with slopes $\ln 2$ and $\ln 4$ connected at $k=1$. In the figures, theoretical curves are also plotted for comparison. Since it is difficult to measure $\lambda(k)$ correctly around the transition point $k=1, \Lambda(k)$ shown in Fig. 2(d) is calculated using the theoretical value of $\lambda(1)$ instead of the numerically measured value. $\Lambda(k)$ and $h(k)$ measured in the initial period indicate clear intermittent growth of the moments, whereas values obtained at larger $t$ do not exhibit intermittency.
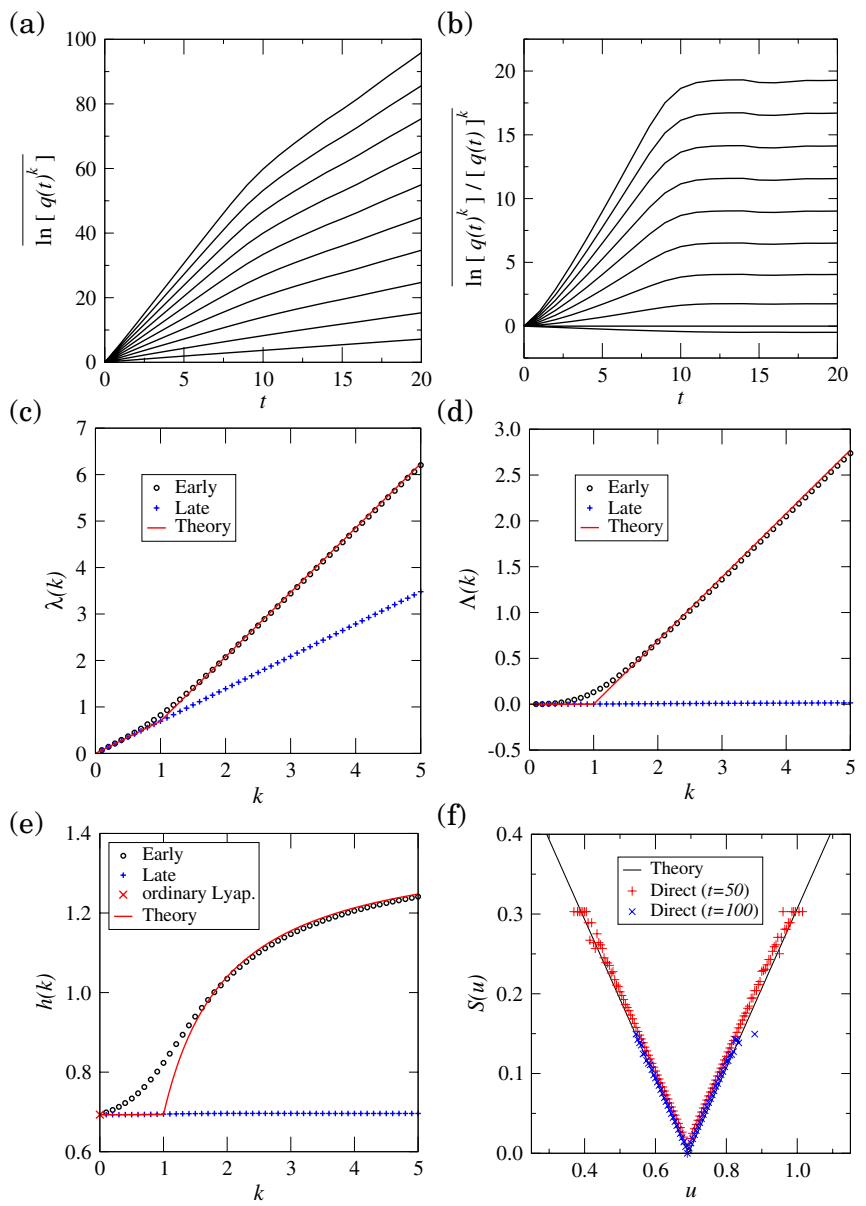

FIG. 2. (Color online) Results for the logistic map. The number of samples is $N=1000$, and the number of averages is $M=1000$. (a),(b) Growth of $\overline{\ln \left[q(t)^{k}\right]}$ and $\overline{\ln \left\{\left[q(t)^{k}\right] /[q(t)]^{k}\right\}}$ at several values of $k$ (the bottom curve corresponds to $k=0.5$, and the top curve to 5.0, with curves in intervals of 0.5 in between). (c),(d),(e) Growth rate $\lambda(k)$, intermittency exponent $\Lambda(k)$, and generalized Lyapunov exponent $h(k)$. Values measured in the initial period and those measured after the onset of relaxation are shown. Theoretical curves are also plotted. (f) Rate function $S(u)$ of the finite-time Lyapunov exponent. Theoretical curve and the results of direct numerical calculations using time intervals of $t=50$ and $t=100$ are plotted.

Figure 2(f) displays the rate function $S(u)$ of the finitetime Lyapunov exponent $u$ which can analytically be calculated as $[6,7]$

$$
S(u)= \begin{cases}\ln 2-u & (u \leq \ln 2), \\ u-\ln 2 & (\ln 2 \leq u \leq \ln 4), \\ \infty & (\ln 4<u) .\end{cases}
$$

Though this $S(u)$ takes its minimum value at the ordinary Lyapunov exponent $u=h=\ln 2$, it does not behave quadratically, but rather linearly around the minimum [6,7], so that the central limit theorem does not hold in this case. In the figure, the above theoretical curve as well as the result of direct numerical calculations using two different time intervals $t=20$ and $t=50$, are shown. 


\section{ESTIMATION OF THE FINITE-SAMPLE EFFECT}

The relaxation of the growth of numerical moments demonstrated in the previous section is due to the use of a finite number of sample trajectories in the numerical calculation. In this section, we analyze the finite-sample effect using the large-deviation formalism.

\section{A. Unobservability of rare events}

By decomposing the time series into rare events, we can estimate the relaxation time of the numerical moments. Though the growth rate $\lambda(k)$ of the $k$ th order moment is determined by Eq. (21) from the rate function $S(u)$ in the large sample limit, in a finite sample set, the probability to observe the dominating event with $u=u^{*}(k)$ decreases exponentially with time, and it eventually becomes unobservable. The number $N(u, t)$ of the event $u$ at time $t$ in a finite sample set of size $N$ is roughly estimated as

$$
N(u, t) \simeq N P(u, t) \sim N \exp \{-t S(u)\} .
$$

In actual numerical calculations, if this $N(u, t)$ is larger than 1 , the event $u$ would likely be observed, whereas if $N(u, t)$ is smaller than 1 , such an event would most likely not be observed. The time $t(u)$ at which the event $u$ becomes unobservable is roughly determined from the condition $N(u, t)$ $=1$ as

$$
t(u)=\frac{1}{S(u)} \ln N
$$

Since the $k$ th order moment is dominated by the event $u^{*}(k)$ satisfying the Legendre condition (20), it would no longer grow exponentially after

$$
t_{\text {sat }}(k)=t\left(u^{*}(k)\right)=\frac{\ln N}{S\left(u^{*}(k)\right)},
$$

at which the event $u^{*}(k)$ becomes unobservable. Since $S(u)$ is a convex function and $u^{*}(k)$ is an increasing function of $k$, higher-order moments relax earlier.

\section{B. Growth law after relaxation}

How do the numerical moments grow after the finitesample relaxation? When $t>t_{\text {sat }}(k)$, the moment $\left[q(t)^{k}\right]$ is no longer dominated by the event $u^{*}(k)$. However, we still expect that the moment is roughly determined by the event with the maximal finite-time Lyapunov exponent $u^{\dagger}(t)$, that can be observed at time $t$ in a finite sample set of size $N$. Such an event can be estimated by inverting the unobservability condition (39) as

$$
u^{\dagger}(t) \simeq S^{-1}\left(\frac{\ln N}{t}\right) .
$$

Thus, after relaxation, the numerical moment $\left[q(t)^{k}\right]$ would roughly behave as

$$
\left[q(t)^{k}\right] \simeq \exp \left\{k u^{\dagger}(t)-S\left(u^{\dagger}(t)\right)\right\} t=\exp \left\{k u^{\dagger}(t) t-\ln N\right\},
$$

and the relative moment after relaxation would be

$$
\frac{\left[q(t)^{k}\right]}{[q(t)]^{k}} \simeq \exp \{(k-1) \ln N\} .
$$

Since $u^{\dagger}(t)$ does not depend on $k$, the growth exponent $\lambda(k)$ depends linearly on $k$, and the intermittency exponent $\Lambda(k)$ vanishes after the finite-sample relaxation. Thus, intermittent growth of the moments would be suppressed, and the relative moments would scale logarithmically with the sample size $N$.

\section{Universal $t^{1 / 2}$ stretched-exponential law}

From Eq. (41), the maximal event $u^{\dagger}(t)$ that can be observed at large $t$ is determined by the behavior of $S(u)$ near its minimum at $u=h$. If the central limit theorem holds for $\eta(t), S(u)$ can generally be approximated quadratically as in Eq. (23) around $u=h$. Thus, $u^{\dagger}(t)$ at sufficiently large $t$ can be approximated as

$$
u^{\dagger}(t) \simeq h+\sqrt{\frac{2 \ln N}{S^{\prime \prime}(h)}} t^{-1 / 2}
$$

for $u^{\dagger}(t)>h$. By inserting this expression into Eq. (42), we can estimate the asymptotic behavior of the $k$ th moment at large $t$ as

$$
\left[q(t)^{k}\right] \simeq \exp \left\{k h t+k \sqrt{\frac{2 \ln N}{S^{\prime \prime}(h)}} t^{1 / 2}-\ln N\right\} .
$$

Thus, after relaxation, we would obtain a growth law of the $k$ th moment that contains a stretched-exponential component whose argument is proportional to $t^{1 / 2}$, in addition to the purely exponential component $\exp (k h t)$ determined by the ordinary Lyapunov exponent, $h$. We verify this estimate below using the modified Bernoulli map, and using the Duffing model in the next section.

As we mentioned previously, the distribution of the expansion rate of chaotic dynamical systems may not always obey the central limit theorem. In such cases, $S(u)$ cannot be approximated by a quadratic curve, and the moments could obey some different asymptotic growth laws after relaxation, as we demonstrate below for the logistic map, and for the Lorenz model in the next section.

\section{Modified Bernoulli map}

We first verify our estimates for the modified Bernoulli map. In this case, the central limit theorem holds, so that $S(u)$ can be approximated by a quadratic curve around its minimum as in Eq. (23). Therefore, we expect that the universal $t^{1 / 2}$ stretched-exponential growth Eq. (45) can be observed.

Figure 3(a) shows numerical relative moments at several values of $k$ in a longer time scale than that of Fig. 1(b). Except for the region of small $t$, the growth of each relative moment is suppressed. From Eq. (43), if we rescale the logarithm of the relative moment

$$
\frac{1}{k-1} \ln \frac{\left[q(t)^{k}\right]}{[q(t)]^{k}} \simeq \ln N
$$

would hold, so that different curves corresponding to different values of $k$ would collapse to a single universal curve that 

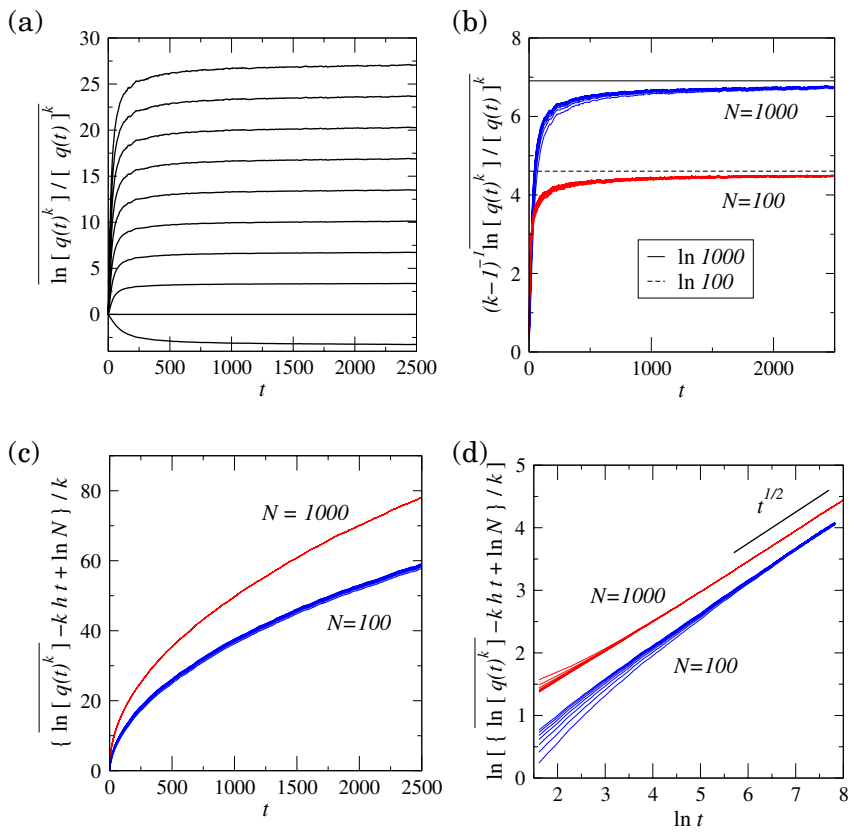

FIG. 3. (Color online) Results of the modified Bernoulli map. (a) Growth of $\ln \left[q(t)^{k}\right] /\{[q(t)]\}^{k}$ obtained numerically at several values of $k$ (the bottom curve corresponds to $k=0.5$, and the top curve to 5.0, with curves in intervals of 0.5 in between) using $N$ $=1000$ samples. (b) Rescaled relative moments obtained using $N$ $=100$ and $N=1000$ samples at $2.0 \leq k \leq 5.0$ with intervals of 0.5 . (c) Rescaled moments obtained using $N=100$ and $N=1000$ samples at $2.0 \leq k \leq 5.0$ with intervals of 0.5 . (d) Double-logarithmic plot of the rescaled moments shown in (c). Theoretically expected stretched-exponential law with $t^{1 / 2}$ is also shown. The number of averages is $M=1000$.

converges to $\ln N$. As shown in Fig. 3(b), the curves corresponding to different values of $k(2.0 \leq k \leq 5.0)$ actually collapse, with the asymptotic value close to the theoretical value $\ln N$ for $N=100$ and $N=1000$. Thus, Eq. (43) seems to be valid in this case.

According to Eq. (45), if we rescale the logarithm of each moment

$$
\frac{\ln \left[q(t)^{k}\right]-k h t+\ln N}{k} \simeq \sqrt{\frac{\ln N}{S^{\prime \prime}(h)}} t^{1 / 2} \propto t^{1 / 2}
$$

would hold. From Fig. 3(c), we can confirm that the curves corresponding to different values of $k(2.0 \leq k \leq 5.0)$ actually collapse to a single universal curve for each value of $N$. Furthermore, from Fig. 3(d), we can confirm that these universal curves approach the asymptotic $t^{1 / 2}$ stretchedexponential law, as we expected.

\section{E. Logistic map}

As we mentioned previously, the central limit theorem does not hold in this case, and the rate function $S(u)$ is given by Eq. (37). Using this expression, the maximal finite-time Lyapunov exponent $u^{\dagger}(t)$ that can be observed at time $t$ after relaxation is estimated as (a)
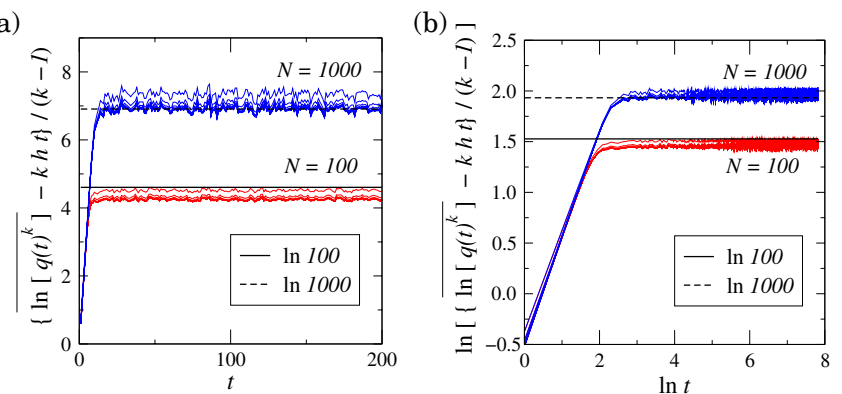

FIG. 4. (Color online) Results of the Logistic map. (a) Rescaled numerical moments at $2.0 \leq k \leq 5.0$ with curves in intervals of 0.5 in between obtained using $N=100$ and $N=1000$. (b) Doublelogarithmic plot of the rescaled numerical moments shown in (a). The number of averages is $M=50$.

$$
u^{\dagger}(t) \simeq h+\frac{\ln N}{t}
$$

so that the growth law of the $k$ th moment after relaxation would be

$$
\left[q(t)^{k}\right] \simeq \exp [k h t+(k-1) \ln N] .
$$

Therefore, if we rescale the numerical moment, the following relation would hold:

$$
\frac{\ln \left[q(t)^{k}\right]-k h t}{k-1} \simeq \ln N .
$$

Figures 4(a) and 4(b) display the rescaled numerical moments at several values of $k(2.0 \leq k \leq 5.0)$ in normal and in double-logarithmic scales for $N=100$ and $N=1000$. As can be seen, different curves corresponding to different values of $k$ collapse to a single universal curve, which converge to a constant value close to $\ln N$ for each value of $N$. Thus, our estimate of the growth law of the relaxed moments appears to be valid also in this case.

\section{CONTINUOUS DYNAMICAL SYSTEMS}

In this section, we demonstrate that the same argument can also be applied to continuous dynamical systems.

\section{A. Growth of the perturbation}

Let us consider a continuous chaotic dynamical system

$$
\dot{\mathbf{x}}(t)=\mathbf{F}(\mathbf{x}(t))
$$

where $\mathbf{x}$ represents the state vector and $\mathbf{F}$ its dynamics. The time $t$ is now a continuous variable. A small perturbation $\mathbf{q}(t)$ to the original orbit $\mathbf{x}(t)$ obeys a linearized equation

$$
\dot{\mathbf{q}}(t)=J_{F}(\mathbf{x}(t)) \mathbf{q}(t) \text {. }
$$

We focus on the direction with the largest expansion rate, and consider the amplitude of the perturbation $q(t)=|\mathbf{q}(t)|$. The instantaneous expansion rate is defined as 


$$
\eta(t)=\lim _{\Delta t \rightarrow 0} \frac{1}{\Delta t} \ln \frac{q(t+\Delta t)}{q(t)},
$$

in a similarly way to Eq. (5) [10]. This $\eta(t)$ is a randomly fluctuating quantity due to the chaotic dynamics of the orbit $\mathbf{x}(t) \cdot q(t)$ obeys

$$
\dot{q}(t)=\eta(t) q(t),
$$

which can be considered as a Langevin equation. The stochastic variable $q(t)$ is driven multiplicatively by the chaotic noise $\eta(t)$, which is generally colored and non-Gaussian. Long-time average of $\eta(t)$ gives the ordinary Lyapunov exponent $h$. The moment $\left\langle q(t)^{k}\right\rangle$ typically grows exponentially as

$$
\left\langle q(t)^{k}\right\rangle \simeq \exp \{\lambda(k) t\}
$$

whose growth rate $\lambda(k)$ is generally a nonlinear function of $k$. The large-deviation argument also holds by simply modifying Eq. (17) as

$$
q(t)=q(0) \exp (u t), \quad u=\frac{1}{t} \int_{s=0}^{t-1} \eta(s) d s .
$$

Therefore, the growth law of the $k$ th moment before relaxation is given by Eq. (19), and that after relaxation would be given by Eq. (42), where $t$ is now regarded as a continuous variable. Especially, when $\eta(t)$ obeys the central limit theorem, $S(u)$ can be approximated by a quadratic curve around its minimum. In that case, we expect that the asymptotic universal post-relaxation growth law (45) holds also in the continuous case.

\section{B. Duffing model}

Let us consider the Duffing model [3]

$$
\begin{gathered}
\dot{x}(t)=v, \\
\dot{v}(t)=-\gamma v-\omega^{2} x-x^{3}+B \sin t,
\end{gathered}
$$

as the first example. By choosing the parameters at $\gamma=0.1$, $\omega^{2}=0.1$, and $B=8.0$, this model exhibits typical chaotic behavior, whose ordinary Lyapunov exponent is $h \simeq 0.097$. We consider a small perturbation $(\delta x, \delta v)$ to the orbit $(x, v)$ and focus on its amplitude $q(t)=\sqrt{\delta x(t)^{2}+\delta v(t)^{2}}$. As in the case of the modified Bernoulli map, it was not necessary to renormalize the perturbation in the numerical calculations. Figures 5(a) and 5(b) display the growth of the moments and the relative moments at several values of $k$ obtained numerically using $N=1000$ samples and $M=1000$ averages. Again, the intermittent exponential growth of the moments can only be observed initially. After some time, the growth law of each moment undergoes a change, and each relative moment stops growing. As shown in Fig. 5(c), the rate function $S(u)$ of the finite-time Lyapunov exponent $u$ can be approximated by a quadratic curve around its minimum. Therefore, as in the case of the modified Bernoulli map, we expect that Eq. (45) holds, and that the moments exhibit universal $t^{1 / 2}$ stretchedexponential growth after relaxation. This can be confirmed in
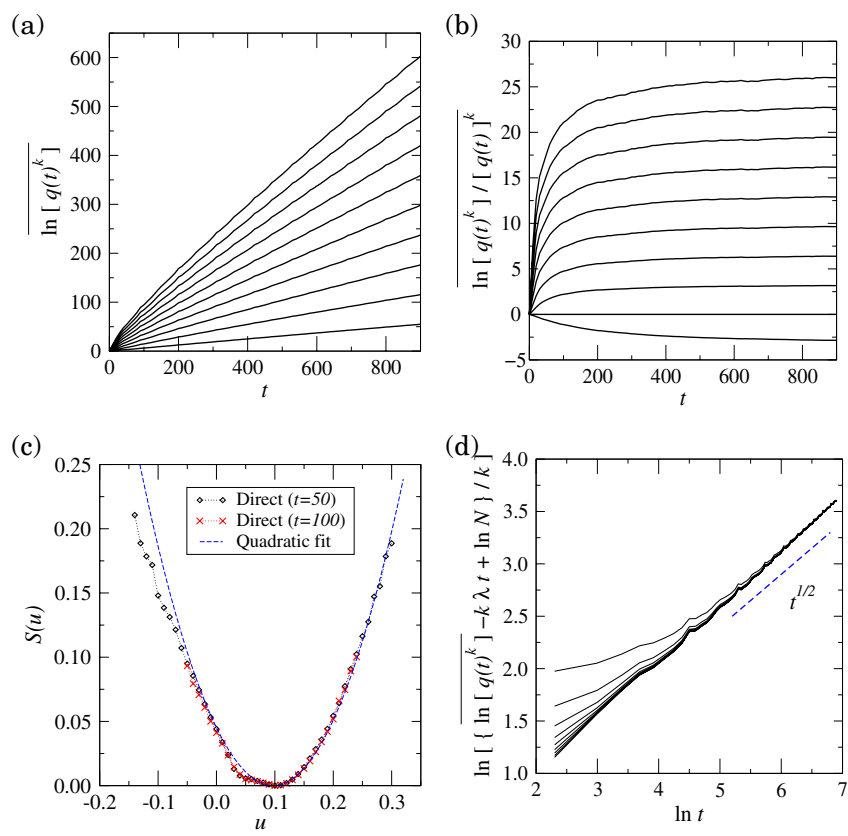

FIG. 5. (Color online) Results for the Duffing model. (a),(b) Growth of $\overline{\ln \left[q(t)^{k}\right]}$, and $\overline{\ln \left\{\left[q(t)^{k}\right] /([q(t)])^{k}\right\}}$. (c) Rate function $S(u)$ obtained by direct numerical simulations using time intervals of $t$ $=50$ and $t=100$. Quadratic fit around the infimum is also plotted. (d) Rescaled moments shown in double-logarithmic scales. The number of samples is $N=1000$ and the number of averages is $M=100$.

Fig. 5(d), where the moments at several values of $k(1.0$ $\leq k \leq 5.0$ with intervals of 0.5 ) plotted in double-logarithmic scales converges to a single universal curve proportional to $t^{1 / 2}$ as $t$ becomes large.

\section{Lorenz model}

As the second example, we consider the chaotic Lorenz model

$$
\begin{gathered}
\dot{x}=p(-x+y), \\
\dot{y}=-x z+q x-y, \\
\dot{z}=x y-r z .
\end{gathered}
$$

We set the parameters at $p=10, q=28$, and $r=8 / 3$, with which the ordinary Lyapunov exponent takes $h \simeq 0.92$. We focus on the amplitude of the linearized perturbation vector $(\delta x, \delta y, \delta z)$, i.e., $q(t)=\sqrt{\delta x(t)^{2}+\delta y(t)^{2}+\delta z(t)^{2}}$. It was not necessary to renormalize the perturbation in the numerical calculations. Figures 6(a) and 6(b) display the growth of the moments and the relative moments obtained numerically using $N=1000$ samples and $M=1000$ averages. As before, the intermittent exponential growth of the moments can be observed only for small $t$. After that, the growth law of each moment changes suddenly, and each relative moment stops growing. Though they exhibit temporal oscillations that reflect the oscillatory dynamics of the Lorenz model, their behavior is qualitatively similar to the previous cases. As shown in Fig. 6(c), the rate function $S(u)$ of the finite-time 
(a)

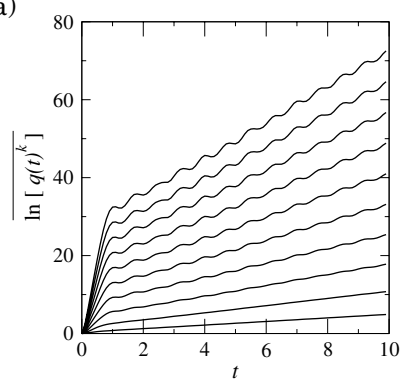

(c)

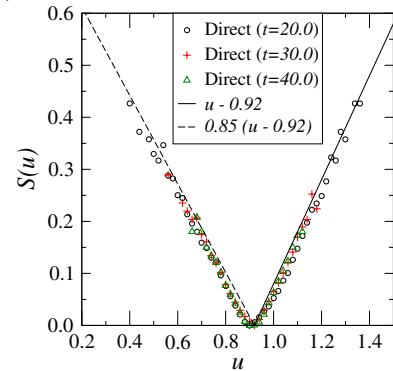

(b)

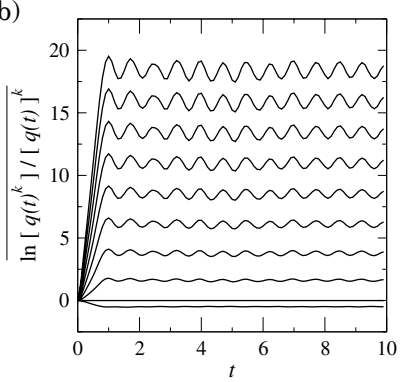

(d)

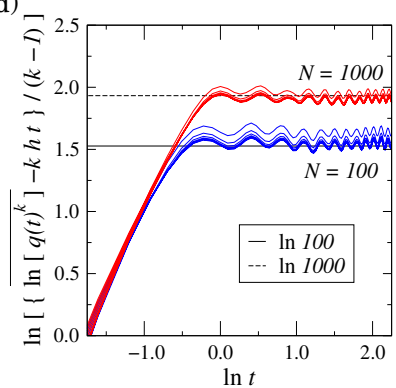

FIG. 6. (Color online) Results for the Lorenz model. (a),(b) Growth of $\overline{\ln \left[q(t)^{k}\right]}$, and $\overline{\ln \left\{\left[q(t)^{k}\right] /([q(t)])^{k}\right\}}$. (c) Rate function $S(u)$ obtained by direct numerical simulations using time intervals of $t$ $=20,30$, and 40. Linear fit around the infimum is also plotted. (d) Rescaled moments shown in double-logarithmic scales. The sample number is $N=100$ or $N=1000$, and the number of averages is $M$ $=1000$.

Lyapunov exponent $u$ does not behave quadratically but rather linearly around its minimum, similarly to the case of the logistic map. They can be fitted by straight lines around the minimum $S(u)=u-h(u>h)$ or $S(u)=0.85(h-u)$ with $h$ $=0.92$, as shown in the figure. Thus, in the same way as the logistic map, we expect Eq. (50) to hold. Figure 6(d) shows the rescaled moments at several values of $k(2.0 \leq k \leq 5.0$ with intervals of 0.5 ) obtained using two different sample sizes $N=100$ and $N=1000$. We can confirm that the curves corresponding to different values of $k$ collapse to a single universal curve that asymptotically approaches the constant $\ln N$ for each $N$.

\section{SUMMARY}

We demonstrated that the growth of the moments of a small perturbation in chaotic dynamical systems suffers from

strong finite-sample effect. The relaxation of growth is attributed to the unobservability of dominant rare events in a finite sample set. Using the large-deviation formalism of chaotic expansion rates, we estimated the relaxation time of the growth, and derived the post-relaxation growth law. Due to the finite-sample effect, all generalized Lyapunov exponents with $k>0$ eventually die out, and only the ordinary Lyapunov exponent corresponding to the $k \rightarrow 0$ limit, the one most insensitive to the fluctuations in the expansion rates [21], survives. However, we demonstrated that each moment still obeys a universal growth law even after the relaxation, which reflects the statistics of the chaotic expansion rates, hence provides us with some physical information on the fluctuating expansion rates. We verified our estimates numerically using chaotic maps and chaotic ordinary differential equations as examples.

In Ref. [22], dynamics of the distance between two trajectories is considered in the fully nonlinear setting, which initially grows exponentially and later reaches a saturation value determined by the size of the chaotic attractor. In contrast, we consider only the linearized evolution of the perturbation in this paper, and still find relaxation of the moment growth due to the finite-sample effect. In Ref. [8], interesting "sporadicity" of intermittent chaotic systems is reported, which leads to stretched-exponential growth of the perturbation in such systems. In this paper, we also observe stretched-exponential growth of the higher order moments of the perturbation for the modified Bernoulli map and for the Duffing model, due to the unobservability of rare events in finite samples, even though the models we treated in our manuscript are not "sporadic" themselves.

It is well known that the finiteness of the sample set significantly affects the numerical estimation of statistical quantities in random multiplicative processes [23]. As we discussed in this paper, it is typical in systems having largedeviation structure, such as the generalized Lyapunov exponents discussed in this paper, and the generalized fractal dimensions of the multifractal measures [24,25]. Our simple argument presented in this paper can be extended to other physical systems with large-deviation structures, where the unobservability of rare fluctuations controls the finite-sample effect. Generalization in this direction is now under progress.

\section{ACKNOWLEDGMENTS}

H.N. thanks Y. Kawamura and K. Arai for useful comments.
[1] H. Fujisaka, Prog. Theor. Phys. 70, 1264 (1983); 71, 513 (1984)

[2] C. Beck and F. Schlögl, Thermodynamics of Chaotic Systems (Cambridge University Press, Cambridge, 1993).

[3] E. Ott, Chaos in Dynamical Systems (Cambridge University Press, Cambridge, 1994).

[4] T. Bohr, M. H. Jensen, G. Paladin, and A. Vulpiani, Dynamical Systems Approach to Turbulence (Cambridge University Press,
Cambridge, 1998).

[5] M. Sano, S. Sato, and Y. Sawada, Prog. Theor. Phys. 76, 945 (1986).

[6] P. Grassberger, R. Badii, and A. Politi, J. Stat. Phys. 51, 135 (1988).

[7] H. Hata, T. Horita, H. Mori, T. Morita, and K. Tomita, Prog. Theor. Phys. 80, 809 (1988).

[8] P. Gaspard and X.-J. Wang, Proc. Natl. Acad. Sci. U.S.A. 85, 
4591 (1988); X.-J. Wang, Phys. Rev. A 39, 3214 (1989); 40, 6647 (1989).

[9] A. S. Pikovsky, Phys. Lett. A 165, 33 (1992); A. Pikovsky, M. Rosenblum, and J. Kurths, Synchronization (Cambridge University Press, Cambridge, England, 2001).

[10] B. Eckhardt and D. Yao, Physica D 65, 100 (1993).

[11] V. Mehra and R. Ramaswamy, Phys. Rev. E 53, 3420 (1996).

[12] A. Prasad and R. Ramaswamy, Phys. Rev. E 60, 2761 (1999).

[13] C. Anteneodo, Phys. Rev. E 69, 016207 (2004).

[14] Y. Oono, Prog. Theor. Phys. Suppl. 99, 165 (1989).

[15] R. S. Ellis, Entropy, Large Deviations, and Statistical Mechanics (Springer, New York, 1985); F. den Hollander, Large Deviations (American Mathematical Society, City, 2000).
[16] A. S. Mikhailov, Phys. Rep. 184, 307 (1989).

[17] A. Mikhailov, Physica A 188, 367 (1992).

[18] D. Zanette and A. Mikhailov, Phys. Rev. E 50, 1638 (1994).

[19] A. S. Mikhailov and A. Yu. Loskutov, Foundations of Synergetics II (Springer, Berlin, 1996).

[20] H. Nakao and A. S. Mikhailov, Chaos 13, 953 (2003).

[21] P. Bergé, Y. Pomeau, and Ch. Vidal, Order Within Chaos (Wiley, New York, 1986).

[22] C. Nicolis and G. Nicolis, Phys. Rev. A 43, 5720 (1991).

[23] S. Redner, Am. J. Phys. 58, 267 (1990).

[24] P. Grassberger, Phys. Lett. A 128, 369 (1988).

[25] A. J. Roberts and A. Cronin, Physica A 233, 867 (1996); A. J. Roberts, e-print nlin.PS/0512014 (unpublished). 